\title{
CUBIC InGaN GROWN BY MOCVD
}

\author{
J.B. Li, Hui Yang, L.X. Zheng, D.P. Xu, Y.T. Wang \\ National Research Center for Opto-electronic Technology, Institute of Semiconductors, \\ CAS, Beijing 100083, China
}

Cite this article as: MRS Internet J. Nitride Semicond. Res. 4S1, G3.25 (1999)

\begin{abstract}
$\underline{\text { Abstract }}$
We report on the growth of high-quality cubic phase InGaN on GaAs by MOCVD. The cubic InGaN layers are grown on cubic GaN buffer layers on GaAs (001) substrates. The surface morphology of the films are mirror-like. The cubic nature of the InGaN films is obtained by Xray diffraction (XRD) measurements. The InGaN layers show strong photoluminescence (PL) at room temperature. Neither emission peak from wurtzite $\mathrm{GaN}$ nor yellow luminescence is observed in our films. The highest In content as determined by XRD is about $17 \%$ with an PL emission wavelength of $450 \mathrm{~nm}$. The FWHM of the cubic InGaN PL peak are $153 \mathrm{meV}$ and 216 $\mathrm{meV}$ for $427 \mathrm{~nm}$ and $450 \mathrm{~nm}$ emissions, respectively. It is found that the In compositions determined from XRD are not in agreement with those estimated from PL measurements. The reasons for this disagreement are discussed.
\end{abstract}

\section{$\underline{\text { Introduction }}$}

The III-V nitrides have long been viewed as a promising system for semiconductors device applications in the blue and ultraviolet (UV) wavelengths in much the same manner that their highly successful As-based and P-based cousins have been exploited in the infrared (IR), red, and green wavelengths. Although most devices have been based on hexagonal films, cubic $\mathrm{GaN}$ is of significant interest [1-5]. Cubic GaN has been grown on substrates that have a cubic lattice, such as GaAs[2,6-8], $\mathrm{Si}[9], 3 \mathrm{C}-\mathrm{Sic}[6-10]$, and $\mathrm{MgO}$ [11]. In our work, Cubic GaN is grown on GaAs substrates. Because GaAs substrates are conductive and easily cleaved, they will simplify the device fabrication process, thus decrease the device cost. So cubic GaN grown on GaAs has an exciting prospect for fabricating blue and geen LED as well as LDs. Now the quality of cubic phase GaN grown on GaAs have reached to a new level. The full width at halfmaximum (FWHM) of room-temperature photoluminescence (PL) for GaN crystal is reported as about $46 \mathrm{meV}$ [12], which approaches that of high quality hexagonal GaN film.

$\mathrm{InGaN}$ is a direct band material which has the band gap energy varying from $1.92 \mathrm{ev}$ for InN to $3.2 \mathrm{ev}$ for cubic GaN. It is a very important material for use as an active layer for bluegreen LED and LDs. However, InGaN is thermally unstable which causes difficulties in growing high quality and high indium concentration InGaN. In our letter, we have grown high quality cubic phase $\operatorname{In}_{\mathrm{x}} \mathrm{Ga}_{1-\mathrm{x}} \mathrm{N}$ on GaAs substrate by MOCVD. The indium composition $\mathrm{x}$ has been achieved as high as 0.17 with an emission wavelength of $450 \mathrm{~nm}$.

\section{Experimental procedure}

Our cubic phase GaN is grown by MOCVD on GaAs substrate, using $\mathrm{H}_{2}$ as carrier gas, high purity $\mathrm{NH}_{3}$ as $\mathrm{N}$ source, triethylgallium (TEGa) as Ga source, and trimethylindium (TMIn) 
as indium source, respectively. The reaction chamber maintains low pressure (76 torr) during growth. A GaN buffer layer was first deposited at $550{ }^{\circ} \mathrm{C}$ for $2 \mathrm{~min}$, then the GaN epilayer with a thickness of $0.7 \mu \mathrm{m}$ was deposited at a temperature of $850{ }^{\circ} \mathrm{C}$. Finally, the InGaN layer with a thickness of $0.3 \mu \mathrm{m}$ was deposited on the GaN layer. X-ray diffraction (XRD) were used to determine the lattice constant and In content of InGaN films. The XRD measurements are performed using a synchrotron radiation with a wavelength of $1.535 \AA$ Room-temperature photoluminescence (PL) measurements were performed on all samples using a computercontrolled PL system. The excitation source is a He-Cd laser which operates at a wavelength of $325 \mathrm{~nm}$ and produces $25 \mathrm{mw}$ of output power. A setup of a monochromator and a photomultiplier is used for PL measurements.

\section{$\underline{\text { Result and Discussion }}$}

Figure 1 shows XRD profiles for several samples $(\omega / 2 \theta$ mode). The positions of $\mathrm{GaN}$ (002) and (004) peaks are at $19.98^{\circ}$ and $42.92^{\circ}$, respectively. The InGaN diffraction peaks of (002) and (004) are separated clearly from GaN diffraction peaks of (002) and (004). It indicates the cubic phase InGaN films have been achieved. No diffraction peaks correspond to hexagonal phase $\mathrm{GaN}$ and InGaN are observed. Different diffraction-peak positions of InGaN indicate different indium composition $\mathrm{x}$ for InGaN films. Using Vegard's law and assuming a lattice constant of InN to be $0.498 \mathrm{~nm}$, we calculated the indium alloy composition $\mathrm{x}$ from the lattice constant of InGaN crystals determined by XRD. The $x$ values for different samples are indicated in Fig. 1. The highest In composition we have achieved here is $17 \%$. The InGaN films show mirror-like surfaces.

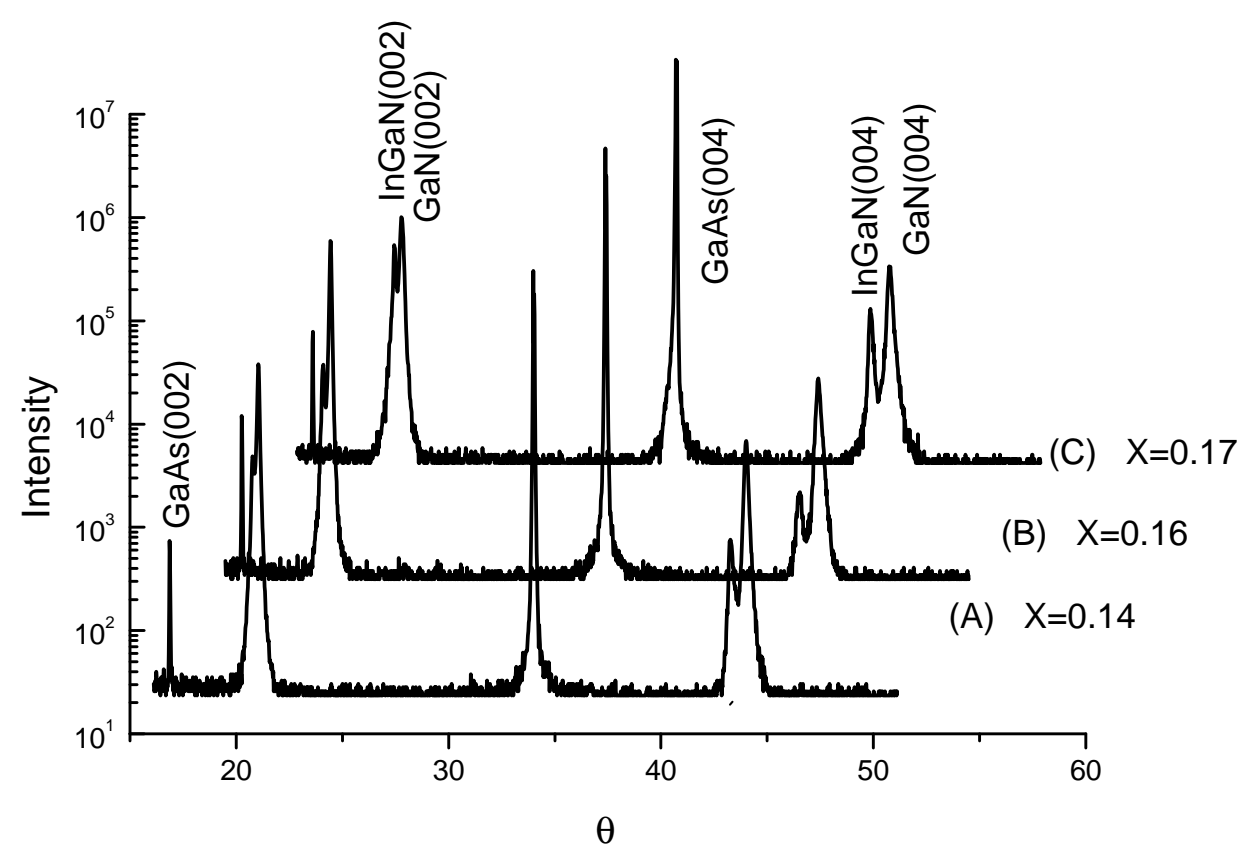

Figure 1. X-ray diffraction profiles ( $\omega / 2 \theta$ mode) for cubic InGaN films. 
Figure 2 shows the room temperature PL spectra for the samples shown in Fig. 1. Except the cubic $\mathrm{GaN}$ band edge emission at $387 \mathrm{~nm}$, in all the spectrum, there is a strong peak at 427 $\mathrm{nm}$ to $450 \mathrm{~nm}$ which we assign to cubic InGaN band edge emission. The FWHM for PL peaks of our InGaN films range from $153 \mathrm{mev}$ to $220 \mathrm{mev}$, with emission wavelength range from $427 \mathrm{~nm}$ to $450 \mathrm{~nm}$. The InGaN peaks in the spectra are quite narrow even compared with hexagonal InGaN. The GaN peak is relatively weak reflecting a strong absorption from the $0.3 \mu \mathrm{m}$ thick InGaN film. The yellow band in our samples are also very weak. The quality of the films becomes better while the indium composition x decreased as indicated by the changes of FWHM.

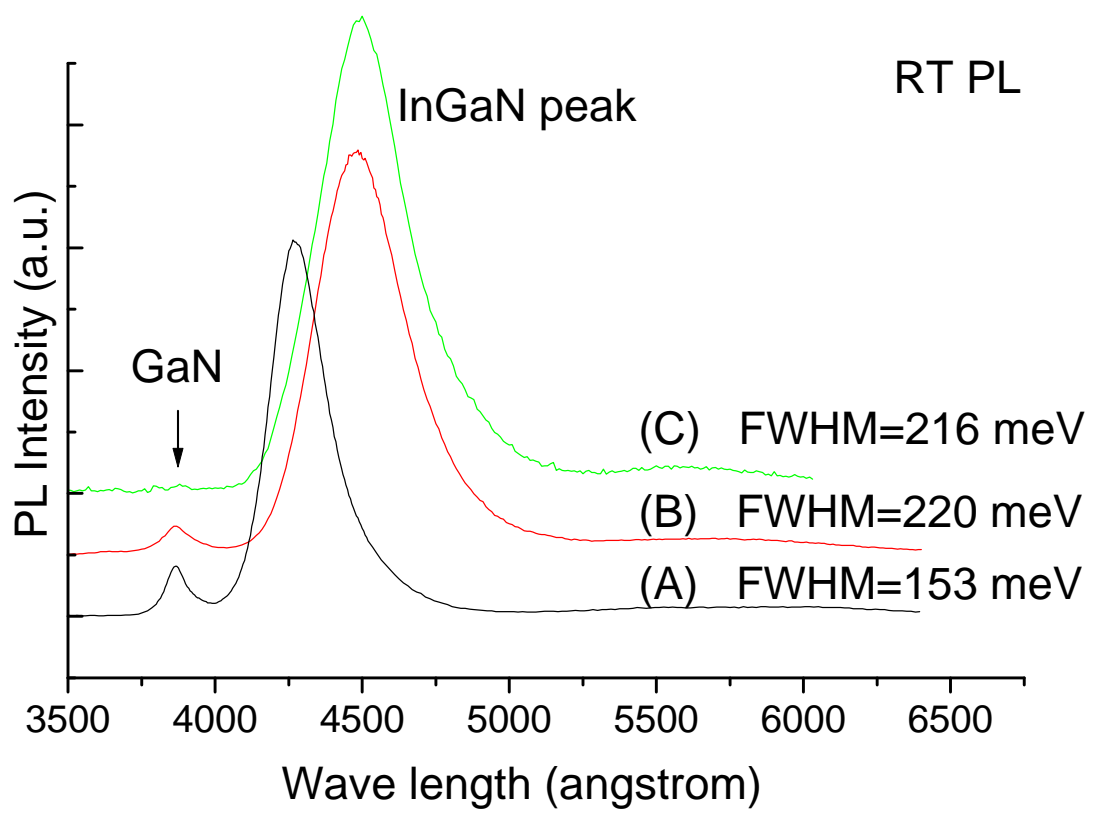

Figure 2. PL spectra of InGaN samples.

We have plotted the PL peak energies of our InGaN samples as a function of the In composition, as shown in Fig. 3. The dashed lines in the figure are linear connections between the two points corresponding to $\mathrm{GaN}(3.2 \mathrm{eV})$ and $\mathrm{InGaN}(1.9 \mathrm{eV}$ to $2.2 \mathrm{eV})$. We can see that the bandgap energy of our InGaN films are well bellow these lines. There are two possibilities for this discrepancy. One is that the In composition is not uniform in the InGaN films. The In composition drawn in the figure is an average value of our sample determined by XRD. It might be not uniform in the samples and much larger than average in some part of the samples. These high In content parts contribute to the PL leading to a longer wavelength than average. In this case, the In composition of the In-rich part might have a value as large as 0.45 . The other possibility is that the In composition is uniform in the films while the bandgap energies of the InGaN are not linearly depend on $\mathrm{x}$. There is bowing behavior in the relationship between the bandgap energy and In composition, as indicated by the solid curve in Fig. 2. Further investigation on this issue are required. 


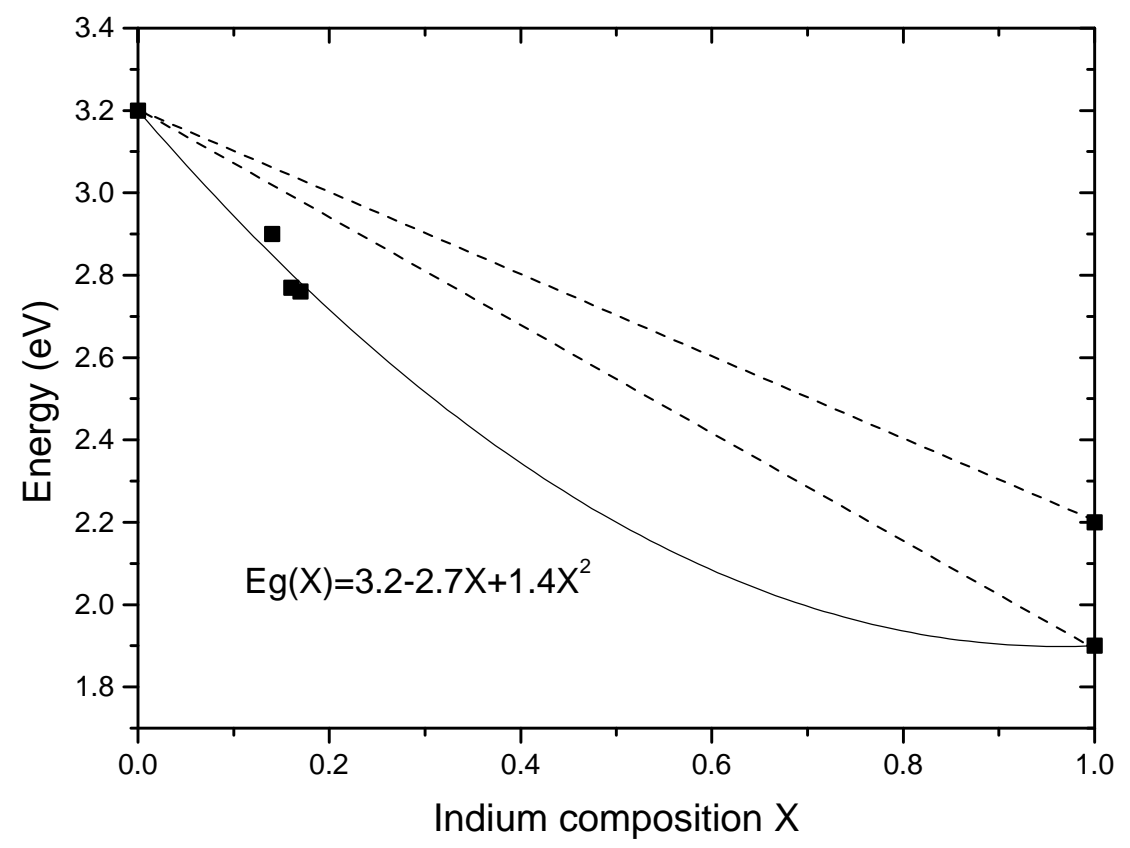

Figure 3. Bandgap energy v.s. indium composition of cubic InGaN. The dashed lines are linear connections between the points corresponding to $\mathrm{GaN}(3.2 \mathrm{eV})$ and $\mathrm{InGaN}(1.9 \mathrm{eV}$ to $2.2 \mathrm{eV}$ ). The solid curve is drawn according to the equation shown in the figure.

\section{$\underline{\text { Summary }}$}

High In content cubic InGaN films have been grown on GaAs substrates by MOCVD. The cubic InGaN and cubic GaN (002) and (004) peaks in XRD profiles can be clearly separated. The lattice constant and In composition of InGaN can then be determined. All the cubic InGaN films show very strong room-temperature PL. The cubic InGaN sample emitting at $450 \mathrm{~nm}$ wavelength has a FWHM of only $220 \mathrm{meV}$, which shows the cubic InGaN film is of high quality.

\section{$\underline{\text { Acknowledgement }}$}

The XRD measurements were carried out in Beijing Synchrotron Radiation facility, Institute of High Energy Physics, CAS, Beijing, China.

\section{$\underline{\text { References }}$}

[1] S. Strite, H.Morkoc, J. Vac. Sci. Technol. B 101227 (1992).

[2] Fujieda, Y. Matsumoto, Jpn. J. Appl. Phys. 30 L1665 (1991).

[3] S. Yoshida, H. Okumura, S. Misawa, E. Sakuma, Surf. Sci. 267, 50(1992).

[4] A. Kikuchi, H. Hoshi, K. Kishino, Jpn. J. Appl. Phys. 33, 688(1994).

[5] S. Bharatan, K. S. Jones, C. R. Abernathy, S. J. Pearton, F. Ren, P. W. Wisk, J. R. Lothian, J. Vac. Sci. Technol A 12, 1094 (1994) 
[6] H. Okumura, S. Yoshida, T. Okahisa, Appl. Phys. Lett. 64, 2997(1994)

[7] J. W. Yang, J. N. Kuznia, Q. C. Chen, M. Asif. Khan, T. George, M. De Graef, S. Mahajan, Appl. Phys. Lett. 67, 3759(1995)

[8] H. Hong, K. Wang, D. Pa Vidis, J. Electron. Mater. 24, 213(1995)

[9] T. Lei, T. D. Moustakas, R. J. Graham, Y. He, S. J. Berkowitz, J. Appl. Phys. 71, 4933(1992)

[10] M. J. Paisley, Z. Sitar, J. B. Posthill, R. F. Davis, J. Vac. Sci. Technol. A 7, 701(1989)

[11] R. C. Powell, N. E. Lee, Y. W. Kim, J. E. Greene, J. Appl. Phys. 73, 189(1989)

[12] Hui Yang, L.X. Zheng, J.B. Li, X.J. Wang, D.P. Xu, Y.T. Wang, and X.W. Hu, submitted to Appl. Phys. Lett. 